\title{
Tuberculosis screening among persons with diabetes mellitus in Pune, India
}

\author{
Vidya Mave ${ }^{1,2^{*}}$, Smita Nimkar $^{1}$, Haridas Prasad ${ }^{1,3}$, Dileep Kadam,3, Sushant Meshram,3, Rahul Lokhande ${ }^{3}$, \\ Nikhil Gupte ${ }^{1,2}$, Divyashri Jain ${ }^{1}$, Amita Gupta ${ }^{1,2}$ and Jonathan E. Golub ${ }^{2}$
}

\begin{abstract}
Background: Diabetes mellitus (DM) increases tuberculosis (TB) risk, and there is increasing concern over the public health implications of the convergence of these two epidemics. Screening for TB among people with DM is now recommended in India.

Methods: People with DM seeking care at a large public sector tertiary care hospital clinic in Pune, India, were screened for TB from June 2015 to May 2016. All consenting people with DM were screened for TB at each clinic visit using a five-item, WHO-recommended questionnaire and those with TB symptoms and/or risk factors were tested for active TB using sputum smear microscopty, Xpert ${ }^{\oplus}$ MTB/RIF and TB culture. Categorical data and continuous variables were summarized using descriptive statistics. The $x^{2}$ test or Wilcoxon rank-sum test was used to ascertain significant associations between categorical and continuous variables, respectively.

Results: Among 630 adults approached for screening, median age was 60 (interquartile range (IQR), 57-64) years and 350 (56\%) were females. Median hemoglobin A1c (HbA1c) was 8.7\% (IQR, 6.7-9.9) and 444 (70.5\%) were poorly controlled DM (HbA1C > 7). Forty-four (7\%) had prior history of TB but the proportion with TB risk factors at screening was low $(<5 \%)$. While $18 \%$ of participants reported any TB symptoms, none of these patients were diagnosed with culture confirmed TB.

Conclusions: Our study failed to yield any active TB cases using a WHO-recommended questionnaire among people with DM. High TB risk populations among people with DM must be identified if TB screening is to be feasible in settings such as India where the DM epidemic continues to rise.
\end{abstract}

Keywords: Tuberculosis, Diabetes mellitus, Risk factors, Screening, India

\section{Background}

Tuberculosis (TB) has recently become the leading cause of mortality related to an infectious agent, even surpassing HIV [1]. While significant gains in global TB control have been achieved, an estimated 10.4 million people developed TB disease and 1.8 million died from the disease in 2015. With increasing burden of non-communicable diseases in low and middle income countries, overlap of conditions, such as diabetes mellitus (DM) and TB has risen, posing additional challenges to disease control and management [2]. Because

\footnotetext{
*Correspondence: vidyamave@gmail.com

'Byramjee-Jeejeebhoy Government Medical College-Johns Hopkins University Clinical Research Site, 1st Floor, Pathology Museum, Jai Prakash Narayan Road, Pune, Maharashtra 411001, India

${ }^{2}$ Johns Hopkins University School of Medicine, Baltimore, MD, USA

Full list of author information is available at the end of the article
}

DM increases TB risk by at least 3-fold and DM burden is increasing, there have been grave concerns that the DM epidemic may slow the decline of global TB incidence and may hinder the goal of achieving the global milestones of a $50 \%$ reduction in $\mathrm{TB}$ incidence and $75 \%$ reduction in TB deaths by 2025 [3-5]. Both the Bali declaration and the World Health Organization (WHO) and the International Union Against Tuberculosis and Lung Disease (IUATLD) joint statement supported a collaborative framework that recommended bidirectional screening including active TB case finding among people with DM $[6,7]$.

In 2015, India alone was estimated to have a staggering 69 million people aged 20-79 years affected with DM [8]. Furthermore, with an estimated over two million new cases annually, India accounts for $18 \%$ of the 
world's estimated TB burden [1]. Therefore, the public health implications of the convergence of these two epidemics in India is paramount, and screening for TB among people with DM may be an important strategy. While several studies have evaluated the burden of DM among TB patients [9-13], data on the burden of TB among people with DM in India are limited [14]. In a multicenter cross-sectional study that screened people with $\mathrm{DM}$ for $\mathrm{TB}$ using a standard $\mathrm{TB}$ symptom-screen approach, 600-950 cases per 100,000 were detected, however whether such a strategy will yield similar results in different regions in India is not known. We present a study that aimed to screen for prevalent TB and TB risk factors among people with DM in western India using WHO recommended TB screening questionnaire [1]. Our study further conducted sputum smear microscopy, gene Xpert and TB culture investigations among those with positive TB symptoms.

\section{Methods}

\section{Study design and population}

We conducted a prospective study of people with DM seeking care at a DM clinic at Byramjee-Jeejeebhoy Medical College-Sassoon General Hospitals (BJMCSGH) in Pune, Maharashtra. BJMC-SGH is a large public sector tertiary care teaching institution that serves approximately 7 million population in the surrounding urban, semi-urban and rural populations. The DM clinic has 1500 adults with DM registered for care and visit the clinic monthly.

\section{Study procedures}

Adults (age $\geq 18$ years) with known DM were consented to participate. Enrolled participants were administered a questionnaire to collect demographics and medical history, including $\mathrm{TB}$ history and TB risk factors. All enrolled participants underwent anthropometric assessments, a baseline random blood sugar test and HbA1c test. A trained research staff administered the WHO symptom screen questionnaire that included screening for cough for longer than 2 weeks, fever, weight loss, loss of appetite, or presence of enlarged lymph glands [1]. Any positive symptoms triggered sputum smear microscopy, gene Xpert and TB culture investigations. Spontaneously expectorated sputum was collected on two occasions. All sputum specimens collected underwent digestion and decontamination by using $\mathrm{NaOH}-\mathrm{NALC}$ method after direct AFB staining, and Xpert ${ }^{\circ}$ MTB/RIF assays were performed. After centrifugation, the sediment was placed on smears for AFB staining and approximately $10 \mu \mathrm{l}$ was inoculated on both Mycobacterial Growth Indicator Tube (MGIT liquid culture) and Lowenstein-Jensen (LJ) slant before incubation. The slants were observed for growth. People with DM were screened using symtpm screen for TB at each visit to the DM clinic over a 12 month period. The Institutional Review Board of Johns Hopkins University and the ethics committee at BJMC-SGH approved the project.

\section{Statistical analysis}

Data were analyzed using STATA (version 13.1). Assuming $0.5 \%$ new TB cases could be detected by symptom screen based on a prior study from India [14], we estimated to detect 4-16 TB cases among $1500 \mathrm{DM}$ patients. The primary outcome of the study was prevalent TB. Secondary outcomes were frequency of TB risk factors among poorly controlled DM (defined as HbA1c $\geq 7 \%$ ) and controlled DM (HbA1c $<7 \%$ ). Baseline and time-updated categorical and continuous variables are summarized using proportions and medians with interquartile range (IQR), respectively, and compared using chi square test, Fisher's exact test or Wilcoxon rank-sum test as appropriate between adults with controlled DM and poorly controlled DM; $P$-values less than 0.05 were deemed statistically significant.

\section{Results}

\section{Study population characteristics}

Of 675 adults with DM who visited the clinic during the study period, 630 consented for TB screening; 350 (56\%) were female, and the median age was 60 years (IQR: $50-65$ ). Median HbA1c was 7.6 (IQR: 6.4-9.3) and $444(70.5 \%)$ were defined as poorly controlled DM. Overall, 32\% had a lower income at Rs. $\leq 10,000$ and $32 \%$ were overweight (body mass index, BMI $>25 \mathrm{~kg} / \mathrm{m} 2$ ). As shown in the Table 1, the demographic and clinical characteristics between controlled and poorly controlled DM are comparable. The majority $(97 \%)$ of people with DM were on oral hypoglycemics, however, compared to those with controlled $\mathrm{DM}$, those with poorly controlled DM were more likelly to be on sulfonylurea ( $55 \%$ vs. $38 \%$ vs. $P=0.0001)$ and metformin ( $82 \%$ vs. $75 \%, p=0.02)$.

\section{TB prevalence and risk factors}

Eigteen percent of participants reported any TB symptoms; $3 \%$ reported cough, $6 \%$ fever, and $9 \%$ weight loss and 9\% night sweats (Table 1). However, no prevalent TB cases were identified. Forty-four (7\%) reported a prior TB history; of these, 5 (11\%) reported prior TB within two years of DM diagnosis, $22(50 \%)$ had a TB diagnosis prior to DM diagnosis and 14 (32\%) had the diagnosis three years after DM diagnosis. Only 13 (2\%) reported a recent contact with a known case of $\mathrm{TB}$. Additional known TB risk factors, smoking (4\%), alcohol abuse $(5 \%)$ lower body mass index $(1 \%)$ were not common and were not statistically different between people with controlled DM and poorly controlled DM. 
Table 1 Demographic and clinical characteristics and risk factors for tuberculosis among people with diabetes mellitus (DM) by glycemic status

\begin{tabular}{|c|c|c|c|c|}
\hline & $\begin{array}{l}\text { Overall } \\
(n=630)\end{array}$ & $\begin{array}{l}\text { Controlled DM } \\
(n=186)\end{array}$ & $\begin{array}{l}\text { Poorly controlled DM } \\
(n=444)\end{array}$ & $P$ value \\
\hline \multicolumn{5}{|l|}{ Demographic characteristics, n (\%) } \\
\hline Female sex & $350(56 \%)$ & $100(54 \%)$ & $250(56 \%)$ & 0.5037 \\
\hline Median age, y (IQR) & $60(50-65)$ & $61(54-66)$ & $60(50-65)$ & 0.00 \\
\hline Marital status & & & & 0.013 \\
\hline Other & $125(20 \%)$ & $33(18 \%)$ & $92(21 \%)$ & \\
\hline Married & $493(78 \%)$ & $145(78 \%)$ & $348(78 \%)$ & \\
\hline Unmaried & $12(2 \%)$ & $8(4 \%)$ & $4(1 \%)$ & \\
\hline Employed & $467(74 \%)$ & $134(72 \%)$ & $333(75 \%)$ & 0.44 \\
\hline Monthly household income & & & & 0.87 \\
\hline$<5000$ & $182(29 \%)$ & $125(28 \%)$ & $57(31 \%)$ & \\
\hline$(5000-10,000)$ & 201 (32\%) & $142(32 \%)$ & $59(32 \%)$ & \\
\hline$>10,000$ & $132(21 \%)$ & $94(21 \%)$ & $38(20 \%)$ & \\
\hline \multicolumn{5}{|l|}{ Diabetes Mellitus characteristics, n (\%) } \\
\hline Median duration of DM, years (IQR) & $6(3-10)$ & $6(3-11)$ & $4(2-7)$ & 0.00 \\
\hline Median HbA1c, (IQR) & $7.6(6.4-9.3)$ & $6.5(6.45-6.65)$ & $8.5(7.5-9.85)$ & 0.00 \\
\hline \multicolumn{5}{|l|}{ Oral hypoglycemics } \\
\hline Sulfonylurea & $314(50 \%)$ & $71(38 \%)$ & $243(55 \%)$ & 0.00015 \\
\hline Metformin & $506(80 \%)$ & $140(75 \%)$ & $366(82 \%)$ & 0.02 \\
\hline Injectable insulin & $21(3 \%)$ & $5(3 \%)$ & $16(4 \%)$ & 0.56 \\
\hline \multicolumn{5}{|l|}{ Tuberculosis risk factors, n (\%) } \\
\hline Past history of TB & $44(7 \%)$ & $17(9 \%)$ & $27(6 \%)$ & 0.16 \\
\hline Recent contact with TB case & $13(2 \%)$ & $7(4 \%)$ & $6(1 \%)$ & 0.07 \\
\hline Tobacco smoking & $24(4 \%)$ & $9(5 \%)$ & $15(3 \%)$ & 0.38 \\
\hline Alchohol abuse & $30(5 \%)$ & $8(4 \%)$ & $22(5 \%)$ & 0.69 \\
\hline Body mass index $\left(\mathrm{kg} / \mathrm{m}^{2}\right)$ & & & & 0.11 \\
\hline Underweight $(<18.5)$ & $8(1 \%)$ & $4(2 \%)$ & $4(1 \%)$ & \\
\hline Normal (18.5-24.9) & $150(24 \%)$ & $34(18 \%)$ & $116(26 \%)$ & \\
\hline Overweight (25-29.9) & $200(32 \%)$ & $60(32 \%)$ & $140(32 \%)$ & \\
\hline \multicolumn{5}{|l|}{ Tuberculosis symptoms, n (\%) } \\
\hline Overall Cough & $66(11 \%)$ & 19 (10\%) & $47(11 \%)$ & \\
\hline Cough $>2$ weeks & $19(3 \%)$ & $8(4 \%)$ & $11(3 \%)$ & 0.24 \\
\hline Fevers & $36(6 \%)$ & $9(5 \%)$ & $27(6 \%)$ & 0.54 \\
\hline Night sweats & $54(9 \%)$ & $14(8 \%)$ & $40(9 \%)$ & 0.52 \\
\hline Weight loss & $59(9 \%)$ & $18(10 \%)$ & $41(9 \%)$ & 0.97 \\
\hline Any of the four symptoms & $111(18) \%$ & 35 (19\%) & $76(17 \%)$ & \\
\hline
\end{tabular}

\section{Discussion}

In our study, the WHO-recommended active TB case finding strategy using a standardized symptom screen did not yield any new prevalent TB cases among patients with DM, even when over two-thirds had poorly controlled DM. Similar findings have been reported from South Africa and Guinea Basseau [15, 16]. In contrast, a prior cross sectional study in India that used a TB symptom-screen approach within routine health services among people with DM detected high rates of prevalent TB (600-950 cases per 100,000) but a large proportion of those newly detected were previously diagnosed and were on treatment prior to screening [14]; only $0.5 \%$ were newly diagnosed $\mathrm{TB}$, confirming that $\mathrm{TB}$ symptom screen yield may be very low for detecting new TB among those with DM. While the joint WHO and IJTLD 
framework's and Bali declaration's recommendation of screening for active TB among people with DM is an important strategy, the feasibility of scaling up a very low yield strategy may not be attractive to national programs. Furthermore, cost-effectiveness of such a strategy is unknown and emerging evidence suggests that symptom screening may poorly predict $\mathrm{TB}$ disease in high risk groups $[17,18]$. Whether enhancing the screening strategy using $\mathrm{Xpert}^{\circ} \mathrm{MTB} / \mathrm{RIF}$ improves yield is yet to be studied.

Whether TB patients with diabetes are less symptomatic has not been supported by prior research that showed more severe or similar clinical presentation of TB among patients with DM $[19,20]$. We found that TB risk factors among people with DM in our setting were fairly low as compared to the general population in India [21]. One interesting finding is that $7 \%$ of the people with DM reported prior TB history, and over $50 \%$ of prior TB was diagnosed before a DM diagnosis. Of these, $11 \%$ reported previous $\mathrm{TB}$ diagnosis within the first 2 years of DM diagnosis [22]. Screening for TB among people with DM is now strongly recommended in India $[6,23]$, though there is a need to identify specific factors among people with DM that increase risk of TB so that screening can be targeted and effective. Understanding the basic epidemiology including incidence and prevalence of TB infection and disease and timing and rate of acquisition or reactivation of TB disease among people with DM is urgently needed to define control strategies.

Our study has several limitations. We only conducted microbiologic investigations on people with symptoms as this is the current recommendation. Secondly, while we included all people with DM consenting to be screened for TB longitudinally, our sample size was small and was restricted to one site. Although our initial estimate was that 4-16 TB cases could be detected among 1500 DM patients, we enrolled only 630 participants. However, even with this sample size, we expected to see 3-16 cases of TB. We did not collect additional information such as lipids, kidney function or blood pressure. Although we did not exclude type $1 \mathrm{DM}$, the majority of patients who visited our DM clinic were type 2 DM patients. Finally, we did not perform radiologic investigations among those with positive TB symptoms, potentially underestimating the TB prevalence. Nonetheless, our study addresses an important question on whether the existing recommendation of TB screening strategy will have utility for India's program where convergence of these two epidemics burdens the health system disproportionately. Furthermore, our study provide a phenotype of DM in this seting.

\section{Conclusions}

Our study demonstrated that a TB screening strategy using the WHO-recommended symptom screen questionnaire may be inadequate among people with DM in India. High TB risk populations among people with DM must be identified if TB screening is to be feasible in settings such as India where the DM epidemic continues to rise. Future studies should confirm our findings; distinguish people with $\mathrm{DM}$ at greatest risk for TB and most feasible diagnostic algorithms for detecting TB among this population; and assess whether targeting persons with DM who are close contacts of TB or who have established risk factors such as longer duration of DM, poor glycemic control, heavy smokers, alcohol users and lower body mass index would be appropriate strategies, as these factors may independently increase TB risk.

\section{Additional file}

Additional file 1: Supplementary file: Raw data of the TB screening in patients with diabetes study. (XLSX $497 \mathrm{~kb}$ )

\section{Abbreviations}

AFB: Acid fast bacilli; DM: Diabetes mellitus; HIV: Human immunodeficiency virus; IUATLD: International Union against tuberculosis and lung disease; TB: Tuberculosis; WHO: World Health Organization

\section{Acknowledgments}

We thank the BJGMC-SGH DM clinic, study staff and study participants for their immense contributions.

Availability of data and materials

All data on which the conclusions of the paper rely are already shown in the table and results section. The raw data used and analysed for the study are in the Additional file 1.

\section{Authors' contributions}

VM, SN, HP, DK, SM, RL,NG, AG conceived the study, VM, SN, HP, DK, ran the study and collected data, VM, NG, DJ, AG, JEG performed data analyses and data interpretation, VM drafted the initial manuscript and all authors assisted in the manuscript preparation and approved the manuscript.

\section{Competing interests}

The authors declare that they have no competing interests.

\section{Consent for publication}

Not applicable as details, images, or videos relating to an individual person has not been shared in the manuscript.

Ethics approval and consent to participate

The Institutional Review Board of Johns Hopkins University (FWA\#00005752) and the ethics committee at BJMC-SGH (FWA\#00005797) approved the project. All study participants provided written informed consent.

\section{Sources of support}

This work was supported by the National Institutes of Health $(\mathrm{NIH}$ 1R01A11097494-01A1 to Golub) and the NIH funded Johns Hopkins Baltimore-Washington-India Clinical Trials Unit for NIAID Networks [U01 Al069497 to VM, NG, AG]. RL was supported by the BJGMC JHU HIV TB Program funded by the Fogarty International Center, National Institutes of Health (NIH) (grant \# D43TW009574). The content of this paper is solely the responsibility of the authors and does not necessary represent the official views of the funders.

\section{Publisher's Note}

Springer Nature remains neutral with regard to jurisdictional claims in published maps and institutional affiliations. 


\section{Author details}

'Byramjee-Jeejeebhoy Government Medical College-Johns Hopkins University Clinical Research Site, 1st Floor, Pathology Museum, Jai Prakash Narayan Road, Pune, Maharashtra 411001, India. ${ }^{2}$ Johns Hopkins University School of Medicine, Baltimore, MD, USA. ${ }^{3}$ Byramjee-Jeejeebhoy Government Medical College, Pune, India.

Received: 25 December 2016 Accepted: 22 May 2017

Published online: 02 June 2017

\section{References}

1. World Helath Organization. Global tuberculosis report 2016. http://www. who.int/tb/publications/global_report/en/. Accessed 20 Oct 2016.

2. Harries AD, Kumar AM, Satyanarayana $S$, et al. Addressing diabetes mellitus as part of the strategy for ending TB. Trans R Soc Trop med Hyg. 2016;110(3):173-9.

3. Odone A, Houben RM, White RG, Lonnroth K. The effect of diabetes and undernutrition trends on reaching 2035 global tuberculosis targets. Lancet Diabetes Endocrinol. 2014;2(9):754-64.

4. Jeon CY, Murray MB. Diabetes mellitus increases the risk of active tuberculosis: a systematic review of 13 observational studies. PLoS med. 2008;5(7):e152.

5. World Health Organization. The End TB Strategy. 2015. www.who.int/tb/ post2015_strategy/en/. Accessed 15 Nov 2016

6. Kapur A, Harries AD, Lonnroth K, Wilson P, Sulistyowati LS. Diabetes and tuberculosis co-epidemic: the Bali declaration. Lancet Diabetes Endocrinol. 2016;4(1):8-10.

7. World Health Organization IUATaLD. Collaborative framework for care and control of tuberculosis and diabetes. 2011.

8. Federation. ID. IDF Diabetes Atlas. 2015

9. Kornfeld H, West K, Kane K, et al. High prevalence and heterogeneity of diabetes in patients with TB in South India: a report from the effects of diabetes on tuberculosis severity (EDOTS) study. Chest. 2016;149(6):1501-8.

10. Nair S, Kumari AK, Subramonianpillai J, et al. High prevalence of undiagnosed diabetes among tuberculosis patients in peripheral health facilities in Kerala. Public Health Action. 2013:3(Suppl 1):S38-42.

11. Viswanathan V, Kumpatla S, Aravindalochanan V, et al. Prevalence of diabetes and pre-diabetes and associated risk factors among tuberculosis patients in India. PLoS One. 2012;7(7):e41367.

12. Screening of patients with tuberculosis for diabetes mellitus in India. Tropical medicine \& international health: TM \& IH 2013; 18(5): 636-45.

13. Samal J. Screening of Tuberculosis patients for possible diabetes mellitus in India: a systematic review. The Egyptian journal of bronchology; 2017; 11: 1-6.

14. Screening of patients with diabetes mellitus for tuberculosis in India. Tropical medicine \& international health: TM \& IH 2013; 18(5): 646-54.

15. Haraldsdottir $T L$, Rudolf $F$, Bjerregaard-Andersen $M$, et al. Diabetes mellitus prevalence in tuberculosis patients and the background population in Guinea-Bissau: a disease burden study from the capital Bissau. Trans R Soc Trop med Hyg. 2015;109(6):400-7.

16. Majumder A, Carroll B, Bhana S, et al. Screening for active tuberculosis in a diabetes mellitus clinic in Soweto, South Africa. The International Journal of Tuberculosis and Lung Disease. 2016;20(7):992-3.

17. Cheng J, Wang L, Zhang H, Xia Y. Diagnostic value of symptom screening for pulmonary tuberculosis in China. PLoS One. 2015;10(5):e0127725.

18. Corbett EL, Zezai A, Cheung YB, et al. Provider-initiated symptom screening for tuberculosis in Zimbabwe: diagnostic value and the effect of HIV status. Bull World Health Organ. 2010;88(1):13-21.

19. Gil-Santana L, Almeida-Junior JL, Oliveira CA, et al. Diabetes is associated with worse clinical presentation in tuberculosis patients from Brazil: a retrospective cohort study. PLoS One. 2016;11(1):e0146876.

20. Alisjahbana B, Sahiratmadja E, Nelwan EJ, et al. The effect of type 2 diabetes mellitus on the presentation and treatment response of pulmonary tuberculosis. Clin Infect dis. 2007;45(4):428-35.

21. Lonnroth K, Jaramillo E, Williams BG, Dye C, Raviglione M. Drivers of tuberculosis epidemics: the role of risk factors and social determinants. Soc Sci Med. 2009;68(12):2240-6.

22. Heo EY, Choi NK, Yang BR, et al. Tuberculosis is frequently diagnosed within 12 months of diabetes mellitus. Int J Tuberc Lung Dis. 2015;19(9):1098-101.

23. Central Tuberculosis division. National framework for joint TB diabetes, 20 Mar 2017. https://tbcindia.nic.in/.../National\%20framework\%20for\%20 joint\%20TB\%20diabetes.pdf

\section{Submit your next manuscript to BioMed Central and we will help you at every step:}

- We accept pre-submission inquiries

- Our selector tool helps you to find the most relevant journal

- We provide round the clock customer support

- Convenient online submission

- Thorough peer review

- Inclusion in PubMed and all major indexing services

- Maximum visibility for your research

Submit your manuscript at www.biomedcentral.com/submit
) BioMed Central 